\title{
POWERFULLY SOLVABLE AND POWERFULLY SIMPLE GROUPS
}

\author{
IKER DE LAS HERAS \\ UNIVERSITY OF THE BASQUE COUNTRY, SPAIN \\ GUNNAR TRAUSTASON \\ UNIVERSITY OF BATH, UK
}

\begin{abstract}
We introduce the notion of a powerfully solvable group. These are powerful groups possessing an abelian series of a special kind. These groups include in particular the class of powerfully nilpotent groups. We will also see that for a certain rich class of powerful groups we can naturally introduce the term powerfully simple group and prove a Jordan-Hölder type theorem that justifies the term.
\end{abstract}

\section{INTRODUCTION}

In this paper $p$ is always an odd prime.

Recall [2] that a finite $p$-group is powerful if $[G, G] \leq G^{p}$. More generally, a subgroup $H$ of $G$ is powerfully embedded in $G$ if $[H, G] \leq H^{p}$.

The following useful property, known as Shalev's Interchange Lemma [3], will be used a number of times in this paper: If $H$ and $K$ are powerfully embedded in $G$ then $\left[H^{p^{i}}, K^{p^{j}}\right]=[H, K]^{p^{i+j}}$.

Another term that we need is a powerful basis. For any powerful $p$-group $G$, there exist generators $a_{1}, \ldots, a_{r}$ such that $G=\left\langle a_{1}\right\rangle \cdots\left\langle a_{r}\right\rangle$ and where $|G|=o\left(a_{1}\right) \cdots o\left(a_{r}\right)$. The number of generators of any given order is an invariant. We say that $G$ is of type $\left(1, \stackrel{r_{1}}{.}, 1,2, \stackrel{r_{2}}{.}, 2, \ldots\right)$ if there are $r_{i}$ generators of order $p^{i}$.

In [5] the notion of powerful nilpotence and powerfully central chain was introduced. If $K \leq$ $H \leq G$, then a chain of subgroups

$$
H=H_{0} \geq H_{1} \geq \cdots \geq H_{n}=K
$$

is powerfully central in $G$ if $\left[H_{i}, G\right] \leq H_{i+1}^{p}$ for $i=0, \ldots, n-1$. A finite $p$-group is said to be powerfully nilpotent if it has a powerfully central series $G=G_{0} \geq G_{1} \geq \cdots \geq G_{n}=1$. The smallest possible length of such a series is then called the powerful nilpotence class of $G$.

Powerful nilpotence leads then naturally to a classification in terms of an ancestry tree and powerful co-class. In [5] it was shown that for every prime $p$ there are finitely many powerfully nilpotent $p$-groups of each powerful co-class, and some general theory was developed for powerfully nilpotent groups. In [6] the powerfully nilpotent groups of maximal powerful class are studied.

In this paper we consider a natural larger class of powerful $p$-groups.

The first author is supported by the Spanish Government grant MTM2017-86802-P, partly with FEDER funds, and by the Basque Government grant IT974-16. He is also supported by a predoctoral grant of the University of the Basque Country. 
Definition. Let $G$ be a finite $p$-group and $K \leq H \leq G$. We say that a chain

$$
H=H_{0} \geq H_{1} \geq \cdots \geq H_{n}=K
$$

is powerfully abelian if $\left[H_{i}, H_{i}\right]=H_{i+1}^{p}$ for $i=0, \ldots, n-1$.

Definition. A finite $p$-group $G$ is powerfully solvable, if there exists a powerfully abelian chain

$$
G=G_{0} \geq G_{1} \geq \cdots \geq G_{n}=1 .
$$

The smallest possible length $n$ is called the powerful derived length of $G$.

The structure of the paper is as follows. In Section 2 we show that all powerful $p$-groups of rank 2 are powerfully solvable and based on the work in [7] we provide a classification of all these groups as well as a closed formula for the number of such groups of order $p^{x}$. In Section 3 we introduce the notion of a powerfully solvable presentation that will be useful later on when going through some classification and calculating growth. In Section 4 we classify all powerful groups of order up to $p^{5}$ and see that these are all powerfully solvable. In Section 5 we discuss the growth of powerfully solvable groups, and various other classes of powerful groups, that are of exponent $p^{2}$. In Section 6 we consider the rich class $\mathcal{P}$ of all powerful $p$ groups of type $(2,2, \ldots, 2)$ and see that powerful nilpotence and powerful solvability play a similar role here as nilpotence and solvability for the class of all groups. The notion of a powerfully simple group arises naturally and we are able to prove a Jordan-Hölder like result that justifies the term. Finally in Section 7 we classify all the powerfully simple groups of order $p^{6}$. The number turns out to depend on the prime $p$.

\section{POWERFUL GROUPS OF RANK 2}

It turns out that all powerful $p$-groups of rank 2 are powerfully solvable. In fact something stronger is true.

Proposition 2.1. Let $G$ be a powerful p-group. If $[G, G]$ is cyclic then $G$ is powerfully solvable of powerful derived length at most 2.

Proof As $G$ is powerful we have that $[G, G]=\left\langle g^{p}\right\rangle$ for some $g \in G$. Therefore

$$
G \geq\langle g\rangle \geq 1
$$

is a powerfully abelian chain.

In [7] the powerfully nilpotent groups of rank 2 are classified and a closed formula is given for the number of powerfully nilpotent groups of order $p^{x}$. In fact there is implicitly the following classification of all powerful $p$-groups of rank 2. By Proposition 2.1 we know that these are all powerfully solvable.

\section{Classification of the non-abelian powerful groups of rank 2.}

(I) Semidirect products:

$$
G=\left\langle a, b: a^{p^{n}}=b^{p^{m}}=1,[a, b]=a^{p^{r}}\right\rangle
$$

with $n-r \leq m$ and $1 \leq r \leq n-1$.

(II) Non-semidirect products:

$$
G=\left\langle a, b: a^{p^{n}}=1, b^{p^{m}}=a^{p^{l}},[a, b]=a^{p^{r}}\right\rangle
$$


with $1 \leq r<l \leq n-1$ and $n-r \leq l<m$.

From [7] we also know that a group above is powerfully nilpotent if and only if $r \geq 2$. Thus, it is easy to determine that there are $\left\lfloor\frac{x-1}{2}\right\rfloor$ semidirect products and $\left\lfloor\frac{x-4}{2}\right\rfloor$ non-semidirect products of order $p^{x}$ that are not powerfully nilpotent (here $\lfloor\cdot\rfloor$ stands for the floor function). From this, the discussion above and [7, Proposition 2.2] we also get the following.

Enumeration. For $x \geq 3$, the number of powerful $p$-groups of rank 2 and order $p^{x}$ is

$$
\begin{array}{r}
\frac{x^{3}+12 x^{2}+12 x}{72} \text { if } x \equiv 0 \quad(\bmod 6) \\
\frac{x^{3}+12 x^{2}+3 x-16}{72} \text { if } x \equiv 1 \quad(\bmod 6) \\
\frac{x^{3}+12 x^{2}+12 x-8}{72} \text { if } x \equiv 2 \quad(\bmod 6) \\
\frac{x^{3}+12 x^{2}+3 x}{72} \text { if } x \equiv 3 \quad(\bmod 6) \\
\frac{x^{3}+12 x^{2}+12 x-16}{72} \text { if } x \equiv 4 \quad(\bmod 6) \\
\frac{x^{3}+12 x^{2}+3 x-8}{72} \text { if } x \equiv 5 \quad(\bmod 6) .
\end{array}
$$

\section{Presentations}

Lemma 3.1. Let $G$ be a finite p-group and let $K<H \leq G$ where $[H, H] \leq K^{p}$. If for some positive integer $n$ we have $K^{p^{n}}=H^{p^{n}}$, then there exists $x \in K \backslash H$ such that $x^{p^{n}}=1$.

Proof We prove this by induction on $n$. Suppose first that $n=1$. We will show that for every $j \geq 1$, there exists $x \in K \backslash H$ such that $x^{p} \in H^{p^{j}}$. For $j=1$ this is immediate from the hypothesis, so assume by induction on $j \geq 2$ that we know that $x^{p} \in H^{p^{j-1}}$ for some $x \in K \backslash H$. Then there exists $y \in H^{p^{j-2}}$ such that $x^{p}=y^{p}$. Now, by the Hall-Petresco Identity, as $p>2$, we have

$$
\left(x y^{-1}\right)^{p}=x^{p} y^{-p} c_{2}^{\left(\begin{array}{c}
p \\
2
\end{array}\right)} c_{3}^{\left(\begin{array}{c}
p \\
3
\end{array}\right)} \cdots c_{p}^{\left(\begin{array}{c}
p \\
p
\end{array}\right)}
$$

where $c_{k} \in\left[H^{p^{j-2}}, K,{ }^{k-1}, K\right]$ for $k=2, \ldots, p$. Notice that $K$ is powerful and that $H$ is powerfully embedded in $K$. We can thus use Shalev's Interchange Lemma. Therefore for $2 \leq k \leq p-1$ we have

$$
c_{k}^{\left(\begin{array}{c}
p \\
k
\end{array}\right)} \in\left[H^{p^{j-2}}, K\right]^{p}=[H, K]^{p^{j-1}} \leq H^{p^{j}}
$$

and

$$
c_{p} \in\left[H^{p^{j-2}}, K, K\right]=[H, K, K]^{p^{j-2}} \leq\left[H^{p}, K\right]^{p^{j-2}}=[H, K]^{p^{j-1}} \leq H^{p^{j}} .
$$

Since $x^{p} y^{-p}=1$, we then have $\left(x y^{-1}\right)^{p} \in H^{p^{j}}$. This finishes the inductive step. Taking $j$ such that $H^{p^{j}}=1$ we see that we can pick $x \in K \backslash H$ such that $x^{p}=1$.

Now suppose $n>1$ and that the result holds for smaller values of $n$. If $K^{p^{n-1}}=H^{p^{n-1}}$, then by induction hypothesis we know there exists $x \in K \backslash H$ where $x^{p^{n-1}}=1$ and thus $x^{p^{n}}=1$. We can thus assume that $K^{p^{n-1}} \neq H^{p^{n-1}}$. Now

$$
\left[K^{p^{n-1}}, K^{p^{n-1}}\right] \leq[K, K]^{p^{2 n-2}} \leq H^{p^{2 n-1}} \leq\left(H^{p^{n-1}}\right)^{p} .
$$

Therefore by the induction hypothesis there exists an element $y \in K^{p^{n-1}} \backslash H^{p^{n-1}}$ such that $y^{p}=1$. Since $K$ is powerful we have $y=x^{p^{n-1}}$ for some $x \in K \backslash H$ and then $x^{p^{n}}=y^{p}=1$. 
Theorem 3.2. Let $G$ be a finite p-group of rank $r$ and exponent $p^{e}$ where $G / G^{p^{2}}$ is powerfully solvable. Then $G$ is powerfully solvable. Furthermore, we can choose our generators $a_{1}, a_{2}, \ldots, a_{r}$ such that $|G|=o\left(a_{1}\right) \cdots o\left(a_{r}\right)$ and such that the chain

$$
\begin{array}{lll}
G=\left\langle a_{1}, a_{2}, \ldots, a_{r}\right\rangle & \geq\left\langle a_{1}^{p}, a_{2}, \ldots, a_{r}\right\rangle & \geq \cdots \geq G^{p} \\
G^{p}=\left\langle a_{1}^{p}, a_{2}^{p}, \ldots, a_{r}^{p}\right\rangle & \geq\left\langle a_{1}^{p^{2}}, a_{2}^{p}, \ldots, a_{r}^{p}\right\rangle & \geq \cdots \geq G^{p^{2}} \\
& \vdots & \\
G^{p^{e-1}}=\left\langle a_{1}^{p^{e-1}}, a_{2}^{p^{e-1}}, \ldots, a_{r}^{p^{e-1}}\right\rangle & \geq\left\langle a_{1}^{p^{e}}, a_{2}^{p^{e-1}}, \ldots, a_{r}^{p^{e-1}}\right\rangle \geq \cdots \geq G^{p^{e}}=1
\end{array}
$$

is powerfully abelian.

Proof Suppose, using the fact that $G / G^{p^{2}}$ is powerfully solvable, that $G=K_{0}>K_{1}>\cdots>$ $K_{m}=G^{p^{2}}$ is a chain that is powerfully abelian modulo $G^{p^{2}}$. Notice that $[G, G] \leq K_{1}^{p} G^{p^{2}} \leq G^{p}$ and the group is thus powerful. In particular, we have $\left[G^{p}, G\right] \leq G^{p^{2}}$ and $\left(G^{p}\right)^{p}=G^{p^{2}}$. Therefore $G=K_{0} G^{p} \geq K_{1} G^{p} \geq \cdots \geq K_{m} G^{p}=G^{p}$ is also powerfully abelian. Removing redundant terms and refining if necessary, we get a powerfully abelian chain

$$
G=H_{0}>H_{1}>\cdots>H_{r}=G^{p}
$$

where the factors are of size $p$. Now notice that for $0 \leq i \leq r-1$ and $0 \leq j \leq e$ we have $\left[H_{i}^{p^{j}}, H_{i}^{p^{j}}\right]=\left[H_{i}, H_{i}\right]^{p^{2 j}} \leq H_{i+1}^{p^{j+1}}$. This gives us the powerfully abelian chain we wanted. It remains to see that we can furthermore pick our generators such that $a_{1}, \ldots, a_{r}$ is a powerful basis for $G$. Let us pick our generators of $G$ such that for every $1 \leq i \leq r-1$ we have $H_{i}=$ $\left\langle a_{i+1}, \ldots, a_{r}\right\rangle \boldsymbol{G}^{p}$. If $\boldsymbol{H}_{i}^{p}=\boldsymbol{H}_{i+1}^{p}$ for some $1 \leq i \leq r-1$ then we can know from Lemma 3.1 that we can pick $a_{i+1}$ such that $a_{i+1}^{p}=1$. We can also in that case move the generator in front. We thus have

$$
a_{1}^{p}=\cdots=a_{r-r_{1}}^{p}=1 \text { and } H_{r-r_{1}}^{p}>\cdots>H_{r}^{p}=G^{p},
$$

where $r_{1}=\operatorname{rank}\left(G^{p}\right)$. Now consider the chain

$$
G^{p^{2}}=H_{r-r_{1}}^{p^{2}} \geq \cdots \geq H_{r}^{p^{2}}=G^{p^{3}} .
$$

Again if $H_{i}^{p^{2}}=H_{i+1}^{p^{2}}$, then we know by Lemma 3.1 that we can pick $a_{i+1}$ such that $a_{i+1}^{p^{2}}=1$. Continuing in this manner we see that we can choose our generators such that for $1 \leq i \leq r$ we have $o\left(a_{i}\right)=p^{j}$ where $j$ is the smallest positive integer such that $H_{i-1}^{p^{j}}=H_{i}^{p^{j}}$. Also we have that $\operatorname{rank}\left(G^{p^{i}}\right)$ is then the number of $1 \leq i \leq r$ such that $a_{i}^{p^{j}} \neq 1$. Let $r_{j}$ be the rank of $G^{p^{j}}$. Then $\left(r_{e}=0\right)$

$$
|G|=p^{r_{0}+r_{1}+\cdots+r_{e-1}}=p^{r_{0}-r_{1}} \cdot\left(p^{2}\right)^{r_{1}-r_{2}} \cdots\left(p^{e-1}\right)^{r_{e-1}-r_{e}}=o\left(a_{1}\right) \cdots o\left(a_{r}\right) .
$$

This finishes the proof.

Powerfully solvable presentations. It follows in particular from Theorem 3.2 that a powerfully solvable group of order $p^{n}$ and rank $r$ has a presentation with generators $a_{1}, \ldots, a_{r}$ and relations

$$
a_{1}^{p^{n_{1}}}=1, \ldots, a_{r}^{p_{r}^{n_{r}}}=1
$$

and

$$
\left[a_{j}, a_{i}\right]=a_{1}^{m_{1}(i, j)} \cdots a_{r}^{m_{r}(i, j)}, 1 \leq i<j \leq r,
$$

where all the power indices $m_{k}(i, j)$ are divisible by $p$ and where furthermore $p^{2} \mid m_{k}(i, j)$ whenever $k \leq i$. Notice that $G$ is the largest finite $p$-group satisfying these relations. To see this let $H$ be the largest finite $p$-group satisfying these relations. The group $H / H^{p^{2}}$ is powerfully solvable and thus $H$ is powerfully solvable by Theorem 3.2. In particular $H$ is powerful and therefore $|H| \leq o\left(a_{1}\right) \cdots o\left(a_{r}\right)$. However $G$ is a homomorphic image of $H$ and thus $|H|=o\left(a_{1}\right) \cdots o\left(a_{r}\right)$. Hence $H$ is isomorphic to $G$. 
A presentation with generators $a_{1}, \ldots, a_{r}$ and relations of the form (1) and (2) is called a powerfully solvable presentation. We say that such a presentation is consistent if the presentation determines a group of order $p^{n_{1}} \cdots p^{n_{r}}$.

\section{Classification OF POWERful GROUPS OF ORDER UP TO $p^{5}$}

In this section we will find all powerful $p$-groups of order up to and including $p^{5}$. It turns out that these are all powerfully solvable. We will see later that there are many powerful groups of order $p^{6}$ that are not powerfully solvable. Let us now turn to our task in this section. There are 2 non-abelian groups of order $p^{3}$. The Heisenberg group of exponent $p$ cannot be powerful as it is of exponent $p$. The other group is a semidirect product of a cyclic group of order $p^{2}$ by a cyclic group of order $p$ :

$$
G_{1}=\left\langle a, b: a^{p^{2}}=b^{p}=1,[a, b]=a^{p}\right\rangle .
$$

Notice that this group is powerfully solvable with a powerfully abelian chain $G\rangle\langle a\rangle\rangle 1$. It is however not powerfully nilpotent as $Z(G)^{p}=1$. Adding to this the 3 abelian groups of order $p^{3}$, we see that there are in total 4 powerfully solvable groups of order $p^{3}$.

Before moving on we consider a general setting like in [7] that includes a number of groups that will occur, namely the non-abelian groups of type $(1, \ldots, 1, n)$ where $n$ is an integer greater than 1. Suppose

$$
G=\left\langle a_{1}, \ldots, a_{t}, b\right\rangle
$$

is a powerful group of this type where $a_{i}$ is of order $p$ and $b$ of order $p^{n}$. Notice that $G^{p}=\left\langle b^{p}\right\rangle$ is cyclic and it follows from [5, Corollary 3.3] that $G^{p} \leq Z(G)$. In particular $G$ is nilpotent of class at most 2 and $[G, G]^{p}=\left[G^{p}, G\right]=1$. Next observe that $\Omega_{1}(G)=\left\langle a_{1}, \ldots, a_{r}, b^{p^{n-1}}\right\rangle$ where $\Omega_{1}(G)$ is the subgroup consisting of all elements of order dividing $p$. Thus $[G, G]=\left\langle b^{p^{n-1}}\right\rangle$. Now, consider the vector space $V=\Omega_{1}(G) G^{p} / G^{p}$ over the field $\mathbb{F}_{p}$ of $p$ elements. The commutator operation naturally induces an alternating form on $V$ through

$$
\left(x G^{p}, y G^{p}\right)=\lambda \text { if }[x, y]=b^{\lambda p^{n-1}} .
$$

Without loss of generality we can suppose that our generators have been chosen such that we get the following orthogonal decomposition

$$
V=\left\langle a_{1} G^{p}, a_{2} G^{p}\right\rangle \oplus \cdots \oplus\left\langle a_{2 s-1} G^{p}, a_{2 s} G^{p}\right\rangle \oplus V^{\perp}
$$

where $V^{\perp}=\left\langle a_{2 s+1} G^{p}, \ldots, a_{t} G^{p}\right\rangle$ and $\left(a_{2 i-1} G^{p}, a_{2 i} G^{p}\right)=1$ for $i=1, \ldots, s$. There are now two cases to consider, depending on whether or not $Z(G) \leq \Omega_{n-1}(G)$.

Suppose first that $Z(G) \not \leq \Omega_{n-1}(G)$. This means that $Z(G)$ contains some element $b^{l} u$ with $u \in\left\langle a_{1}, \ldots, a_{r}\right\rangle$ and $0<l<p$. Thus without loss of generality we can assume that $b \in Z(G)$. We thus get a powerful group $G=A(n, t, s)$ with relations

$$
\begin{aligned}
& a_{1}^{p}=\cdots=a_{t}^{p}=b^{p^{n}}=1 ; \\
& {\left[a_{2 i-1}, a_{2 i}\right]=b^{p^{n-1}} \text { for } i=1, \ldots, s ;} \\
& {\left[a_{i}, a_{j}\right]=1 \text { otherwise for } 1 \leq i<j \leq t ;} \\
& {\left[a_{i}, b\right]=1 \text { for } 1 \leq i \leq t .}
\end{aligned}
$$

Notice that we have $\langle b\rangle \leq Z(G)$ and $[G, G] \leq\left\langle b^{p}\right\rangle$ and thus these groups are all powerfully nilpotent, as was observed in [7]. Notice that for a fixed $n \geq 2$ and $t \geq 2$ we get $\lfloor t / 2\rfloor$ such groups.

We then consider the case when $Z(G) \leq \Omega_{n-1}(G)$. Notice first that replacing $b$ by a suitable $b a_{1}^{\alpha_{1}} \cdots a_{2 s}^{\alpha_{2 s}}$, we can assume that $b$ commutes with $a_{1}, \ldots, a_{2 s}$. As $b \notin Z(G)$ we then must 
have $t>2 s$ and similarly, replacing $a_{i}$ by a suitable $a_{2 s+1}^{\alpha_{2 s+1}} \cdots a_{t}^{\alpha_{t}}$, we can pick our generators $a_{2 s+1}, \ldots, a_{t}$ such that $\left[a_{2 s+1}, b\right]=b^{p^{n-1}}$ and $\left[a_{2 s+2}, b\right]=\cdots=\left[a_{t}, b\right]=1$. We thus arrive at a group $G=B(n, t, s)$ satisfying the relations

$$
\begin{aligned}
& a_{1}^{p}=\cdots=a_{t}^{p}=b^{p^{n}}=1 \\
& {\left[a_{2 i-1}, a_{2 i}\right]=b^{p^{n-1}} \text { for } i=1, \ldots, s} \\
& {\left[a_{i}, a_{j}\right]=1 \text { otherwise for } 1 \leq i<j \leq t ;} \\
& {\left[a_{1}, b\right]=\cdots=\left[a_{2 s}, b\right]=\left[a_{2 s+2}, b\right]=\cdots=\left[a_{t}, b\right]=1 \text { for } 1 \leq i \leq t ;} \\
& {\left[a_{2 s+1}, b\right]=b^{p^{n-1}} .}
\end{aligned}
$$

Notice that for a fixed $n \geq 2$ and $t \geq 1$ there are $\lfloor(t+1) / 2\rfloor$ such groups. Notice also that when $n \geq 3$ then the group is powerfully nilpotent as $\left\langle b^{p}\right\rangle \leq Z(G)$ and $[G, G] \leq\left\langle b^{p^{2}}\right\rangle$. For $n=2$ this is not the case but the group is still powerfully solvable as we have a powerfully abelian chain $G>\langle b\rangle>1$ with $[G, G] \leq\left\langle b^{p}\right\rangle$. We are now ready for groups of order $p^{4}$. In the following we will omit writing relations of the form $[x, y]=1$.

Groups of order $p^{4}$. From our analysis of non-abelian groups of rank 2 we get two such groups:

$$
G_{2}=\left\langle a, b: a^{p^{2}}=b^{p^{2}}=1,[a, b]=a^{p}\right\rangle \text { and } G_{3}=\left\langle a, b: a^{p^{3}}=b^{p}=1,[a, b]=a^{p^{2}}\right\rangle .
$$

Here $G_{3}$ is furthermore powerfully nilpotent. The only non-abelian groups apart from these are of type $(1,1,2)$ and from the analysis of such groups above we know there are two groups:

$$
G_{4}=A(2,2,1)=\left\langle a, b, c: a^{p}=b^{p}=c^{p^{2}}=1,[a, b]=c^{p}\right\rangle,
$$

and

$$
G_{5}=B(2,2,0)=\left\langle a, b, c: a^{p}=b^{p}=c^{p^{2}}=1,[a, c]=c^{p}\right\rangle .
$$

Apart from these there are 5 abelian groups and we thus get in total 9 groups.

Groups of order $p^{5}$. Again our analysis of groups of rank 2 and those of type $(1,1,3)$ and $(1,1,1,2)$ gives us the following non-abelian powerfully solvable groups:

$$
\begin{aligned}
& G_{6}=\left\langle a, b: a^{p^{2}}=b^{p^{3}}=1,[a, b]=a^{p}\right\rangle, G_{7}=\left\langle a, b: a^{p^{3}}=b^{p^{2}}=1,[a, b]=a^{p}\right\rangle, \\
& G_{8}=\left\langle a, b: a^{p^{3}}=b^{p^{2}}=1,[a, b]=a^{p^{2}}\right\rangle, G_{9}=\left\langle a, b: a^{p^{4}}=b^{p}=1,[a, b]=a^{p^{3}}\right\rangle,
\end{aligned}
$$

and

$$
\begin{aligned}
& G_{10}=A(3,2,1)=\left\langle a, b, c: a^{p}=b^{p}=c^{p^{3}}=1,[a, b]=c^{p^{2}}\right\rangle ; \\
& G_{11}=B(3,2,0)=\left\langle a, b, c: a^{p}=b^{p}=c^{p^{3}}=1,[a, c]=c^{p^{2}}\right\rangle ; \\
& G_{12}=A(2,3,1)=\left\langle a, b, c, d: a^{p}=b^{p}=c^{p}=d^{p^{2}}=1,[a, b]=d^{p}\right\rangle ; \\
& G_{13}=B(2,3,0)=\left\langle a, b, c, d: a^{p}=b^{b}=c^{p}=c^{p^{2}}=1,[a, b]=d^{p},[c, d]=d^{p}\right\rangle ; \\
& G_{14}=B(2,3,1)=\left\langle a, b, c, d: a^{p}=b^{p}=c^{p}=d^{p^{2}}=1,[a, b]=d^{p},[c, d]=d^{p}\right\rangle .
\end{aligned}
$$

Here $G_{8}, G_{9}, G_{10}, G_{11}, G_{12}$ are furthermore powerfully nilpotent. Apart from these 9 groups, there are 7 abelian groups. We are now only left with the non-abelian groups of type $(1,2,2)$ that will contain a number of different groups and we need to deal with a number of subcases.

Suppose that we have generators $a, b, c$ of orders $p, p^{2}, p^{2}$.

Case 1. $\left(Z(G)^{p} \neq 1\right)$. Notice that we then must have $\left|Z(G)^{p}\right|=p$ as otherwise $G / Z(G)$ is cyclic and thus $G$ abelian. We can assume that $c \in Z(G)$ and that $Z(G)^{p}=\left\langle c^{p}\right\rangle$. Notice also that $[G, G]=\langle[a, b]\rangle$ is cyclic. There are two possibilities. On the one hand, if $[G, G] \leq Z(G)^{p}$, then we can choose our generators so that we get a group with the following presentation:

$$
G_{15}=\left\langle a, b, c: a^{p}=b^{p^{2}}=c^{p^{2}}=1,[a, b]=c^{p}\right\rangle .
$$


On the other hand if $[G, G] \not Z Z(G)^{p}$, it is not difficult to see that we can pick our generators so that we get a group with the presentation

$$
G_{16}=\left\langle a, b, c: a^{p}=b^{p^{2}}=c^{p^{2}}=1,[a, b]=b^{p}\right\rangle .
$$

Notice that both these groups are powerfully solvable and that $G_{15}$ is furthermore powerfully nilpotent.

Case 2. $\left(Z(G)^{p}=1\right.$ and $G / Z(G)$ has rank 2). Then we must have $a \in Z(G)$. It is not difficult to see that in this case we can choose $b, c$ such that $[b, c]=c^{p}$ and we get the powerfully solvable group

$$
G_{17}=\left\langle a, b, c: a^{p}=b^{p^{2}}=c^{p^{2}}=1,[b, c]=c^{p}\right\rangle .
$$

Before considering further cases, we first show that if $Z(G)^{p}=1$ and $G / Z(G)$ has rank 3 , then we must have $[G, G]=G^{p}$. Note that $\left|G^{p}\right|=p^{2}$, so suppose by contradiction, that $\left|G^{\prime}\right|=p$. Observe that $G^{p} \leq Z(G)$, so $G / Z(G)$ is a vector space over $\mathbb{F}_{p}$. Then, the commutator map in $G$ induces a non-degenerate alternating form on $G / Z(G)$, and so $\operatorname{dim}_{\mathbb{F}_{p}}(G / Z(G))$ is even. This is a contradiction since $G / Z(G)$ has rank 3 . We have thus shown that $[G, G]=G^{p}$. In order to distinguish further between different cases, we next turn our attention to $\left[\Omega_{1}(G), G\right]$. Notice that $\Omega_{1}(G)=\langle a\rangle G^{p}$. As $a \notin Z(G)$ either $\left|\left[\Omega_{1}(G), G\right]\right|$ is of size $p$ or $p^{2}$.

Case 3. $\left(Z(G)^{p}=1, G / Z(G)\right.$ of rank 3 and $\left.\left|\left[\Omega_{1}(G), G\right]\right|=p\right)$. Without loss of generality we can assume that $\left[\Omega_{1}(G), G\right]=\left\langle c^{p}\right\rangle$. There are two possibilities. Either $c \in C_{G}\left(\Omega_{1}(G)\right)=C_{G}(a)$ or not. Suppose first that $c \in C_{G}\left(\Omega_{1}(G)\right)$. Then we have $[a, c]=1$, and we can pick $b$ such that $[a, b]=c^{p}$. Replacing $b$ by $b c^{l}$ does not change these relations and thus we can assume that $[b, c]=b^{p \alpha}$ for some $0<\alpha<p$. If we let $\beta$ be the inverse of $\alpha$ modulo $p$ and we replace $a, c$ by $a^{\beta}, c^{\beta}$, then we arrive at a group with presentation

$$
G_{18}=\left\langle a, b, c: a^{p}=b^{p^{2}}=c^{p^{2}}=1,[a, b]=c^{p},[b, c]=b^{p}\right\rangle .
$$

Notice that this is a powerfully solvable group with a powerfully abelian chain $G>\langle b, c\rangle>$ $\langle b\rangle>1$. Suppose now $c \notin C_{G}\left(\Omega_{1}(G)\right)$. Since $\left|\left[\Omega_{1}(G), G\right]\right|=|[a, G]|=p$, it follows that the conjugacy class of $a$ has order $p$, and so $\left|G: C_{G}(a)\right|=p$. Thus, we can pick $b$ such that $b \in C_{G}(a)$ and $[a, b]=1$. Replacing $a$ by a suitable power of $a$ we can suppose that $[a, c]=c^{p}$. As before, replacing $b$ by $b c^{l}$ does not change these relations, so we can also assume $[b, c]=b^{\alpha p}$ for some $0<\alpha<p$. Finally, if we let $\beta$ be the inverse of $\alpha$ modulo $p$ and we replace $c$ by $c^{\beta}$, we arrive at a group with presentation

$$
G_{19}=\left\langle a, b, c: a^{p}=b^{p^{2}}=c^{p^{2}}=1,[a, c]=c^{p},[b, c]=b^{p}\right\rangle .
$$

This group is powerfully solvable with powerfully abelian chain $G>\langle b, c\rangle>\langle b\rangle>1$.

Case 4. $\left(Z(G)^{p}=1, G / Z(G)\right.$ of rank 3 and $\left.\left|\left[\Omega_{1}(G), G\right]\right|=p^{2}\right)$. In this case, commutation with $a$ induces a bijective linear map

$$
\begin{aligned}
F_{a}: G / \Omega_{1}(G) & \longrightarrow G^{p} \\
x \Omega_{1}(G) & \longmapsto[a, x] .
\end{aligned}
$$

Identifying $x \Omega_{1}(G)$ with $x^{p}$, we can think of $F_{a}$ as a linear operator on a two dimensional vector space over $\mathbb{F}_{p}$. Also replacing $b, c$ by a suitable $b a^{r}, c a^{s}$ we can assume throughout that $[b, c]=1$. All the groups are going to be powerfully solvable with powerfully abelian chain $G>\langle b, c\rangle>1$.

Case 4.1. ( $F_{a}$ is a scalar multiplication). Notice that this property still holds if we replace $a$ by any power of $a$ and thus it is independent of what $a$ we pick in $\Omega_{1}(G) \backslash G^{p}$. This is thus a 
characteristic property of $G$. Replacing $a$ with a power of itself we can assume that $F_{a}$ is the identity map. This gives us the group

$$
G_{20}=\left\langle a, b, c: a^{p}=b^{p^{2}}=c^{p^{2}}=1,[a, b]=b^{p},[a, c]=c^{p}\right\rangle .
$$

Case 4.2. ( $F_{a}$ is not a scalar multiplication). Again we see that this is a characteristic property of $G$. We can now pick $b$ and $c$ such that

$$
[a, b]=c^{p},[a, c]=b^{p \alpha} c^{p \beta} .
$$

Notice that the matrix for $F_{a}$ is

$$
\left[\begin{array}{ll}
0 & \alpha \\
1 & \beta
\end{array}\right]
$$

with determinant $-\alpha$. This is an invariant for the given $a$ that does not depend on our choice of $b$ and $c$. If we replace $a$ by $a^{r}$ and $c$ by $c^{r}$ then we get

$$
[a, b]=c^{p},[a, c]=b^{p \alpha r^{2}} c^{p \beta r},
$$

and the new determinant becomes $-\alpha r^{2}$. Pick some fixed $\tau$ such that $-\tau$ is a non-square in $\mathbb{F}_{p}$. With appropriate choice of $r$ we can then assume that the determinant of $F_{a}$ is $-\alpha$ where either $\alpha=-1$ or $\alpha=\tau$. We thus have a group with one of the two presentations

$$
G_{21}(\beta)=\left\langle a, b, c: a^{p}=b^{p^{2}}=c^{p^{2}}=1,[a, b]=c^{p},[a, c]=b^{-1} c^{p \beta},[b, c]=1\right\rangle,
$$

and

$$
G_{22}(\beta)=\left\langle a, b, c: a^{p}=b^{p^{2}}=c^{p^{2}}=1,[a, b]=c^{p},[a, c]=b^{\tau} c^{p \beta},[b, c]=1\right\rangle .
$$

Suppose we pick a different $\bar{b}=b^{r} c^{s}$. Then for $\alpha \in\{-1, \tau\}$ we have

$$
[a, \bar{b}]=[a, b]^{r}[a, c]^{s}=c^{p r}\left(b^{p \alpha} c^{p \beta}\right)^{s}=b^{p s \alpha} c^{p(r+s \beta)}=\bar{c}^{p}
$$

where $\bar{c}=b^{s \alpha} c^{r+s \beta}$. Then

$$
\begin{aligned}
{[a, \bar{c}] } & =[a, b]^{s \alpha}[a, c]^{r+s \beta} \\
& =c^{p s \alpha}\left(b^{p \alpha} c^{p \beta}\right)^{r+s \beta} \\
& =\left(b^{r} c^{s}\right)^{p \alpha} \cdot\left(b^{s \alpha} c^{r+s \beta}\right)^{p \beta} \\
& =\bar{b}^{p \alpha} \bar{c}^{p \beta}
\end{aligned}
$$

This shows that for the given $\alpha \in\{-1, \tau\}$, the constant $\beta \in \mathbb{F}_{p}$ is an invariant and we get $p$ distinct groups $G_{21}(\beta)$ and $p$ distinct groups $G_{22}(\beta)$.

Adding up we have 7 abelian groups and the groups $G_{6}, \ldots, G_{20}, G_{21}(\beta), G_{22}(\beta)$, giving us in total $22+2 p$ groups of order $p^{5}$.

Notice that we have seen that all powerful groups of order up to and including $p^{5}$ are powerfully solvable. Now take a powerful group of order $p^{6}$. Suppose it has a generator $a$ of order $p$, say $G=\langle a, H\rangle$ where $H<G$. Notice that $H$ is then powerful of order $p^{5}$ and thus powerfully solvable. As $[G, G] \leq H^{p}$ we then see that $G$ is powerfully solvable. Thus all powerful groups of order $p^{6}$ are powerfully solvable with the possible exceptions of some groups of type $(2,2,2)$. We will see later that there are a number of groups of type $(2,2,2)$ that are not powerfully solvable. 


\section{GROWTH}

Let $G$ be a powerfully solvable group of order $p^{n}$. From Theorem 3.2 and the discussion in Section 3, we know that we may assume that $G=\left\langle a_{1}, \ldots, a_{y}, a_{y+1}, \ldots, a_{y+x}\right\rangle$ where $o\left(a_{1}\right)=\cdots=$ $o\left(a_{y}\right)=p$ and $o\left(a_{y+1}\right)=\cdots=o\left(a_{y+x}\right)=p^{2}$. Furthermore the generators can be chosen such that $|G|=p^{y+2 x}$ and

$$
\left[a_{j}, a_{i}\right]=a_{i+1}^{p \alpha_{i+1}^{(i, j)}} \cdots a_{y+x}^{p \alpha_{y+x}(i, j)}
$$

for $1 \leq i<j \leq y+x$, where $0 \leq \alpha_{k}(i, j) \leq p-1$ for $k=i+1, \ldots, y+x$. For each such pair $(i, j)$ where $1 \leq i \leq y$ there are $p^{x}$ possible relations for $\left[a_{j}, a_{i}\right]$. There are $y x+\left(\begin{array}{l}y \\ 2\end{array}\right)$ such pairs. On the other hand, for a pair $(i, j)$ where $y+1 \leq i \leq y+x$, for each given $i$ there are $y+x-i$ such pairs and $p^{y+x-i}$ possible relations $\left[a_{j}, a_{i}\right]$. Adding up we see that the number of solvable presentations is $p^{h(x)}$ where

$$
\begin{aligned}
h(x) & =\left(y x+\left(\begin{array}{l}
y \\
2
\end{array}\right)\right) x+1^{2}+2^{2}+\cdots(x-1)^{2} \\
& =\left((n-2 x) x+\left(\begin{array}{c}
n-2 x \\
2
\end{array}\right)\right) x+\frac{x(2 x-1)(x-1)}{6} \\
& =\frac{1}{3} x^{3}-\frac{(2 n-1)}{2} x^{2}+\frac{3 n(n-1)+1}{6} x
\end{aligned}
$$

Thus

$$
h^{\prime}(x)=x^{2}-(2 n-1) x+\frac{3 n(n-1)+1}{6},
$$

whose roots are $\frac{2 n-1}{2}-\sqrt{\frac{1}{2} n^{2}-\frac{n}{2}+\frac{1}{12}}$ and $\frac{2 n-1}{2}+\sqrt{\frac{1}{2} n^{2}-\frac{n}{2}+\frac{1}{12}}$. For large values of $n$ we have that the first root is between 0 and $n / 2$ whereas the latter is greater than $n$. Thus, for large $n$, the largest value of $h$ in the interval between 0 and $n / 2$ is $h(x(n))$ where $x(n)=\frac{2 n-1}{2}-$ $\sqrt{\frac{1}{2} n^{2}-\frac{n}{2}+\frac{1}{12}}$. Now $\lim _{n \rightarrow \infty} x(n) / n=1-\frac{1}{\sqrt{2}}$. Therefore

$$
\left.\lim _{n \rightarrow \infty} \frac{h(x(n)}{n^{3}}=\lim _{n \rightarrow \infty} \frac{1}{3}(x(n) / n)^{3}-(x(n) / n)^{2}+\frac{1}{2}(x(n) / n)\right)=\frac{-1+\sqrt{2}}{6} .
$$

We now argue in a similar way as in [5]. Let $n$ be fixed. For any integer $x$ where $0 \leq x \leq n / 2$, let $\mathcal{P}(n, x)$ be the collection of all powerfully solvable presentations as above. It is not difficult to see that those presentations are consistent and thus the resulting group is of order $p^{n}$ and rank $n-x$. Furthermore $a_{1}^{p}=\cdots=a_{n-2 x}^{p}=1$ and $a_{n-2 x+1}^{p^{2}}=\cdots=a_{n-x}^{p^{2}}=1$. We have just seen that, for large values of $n$, if we pick $x(n)$ such that the number of presentations is maximal then

$$
|\mathcal{P}(n, x(n))|=p^{\alpha n^{3}+o\left(n^{3}\right)}
$$

where $\alpha=\frac{-1+\sqrt{2}}{6}$. Let $\mathcal{P}_{n}$ be the total number of the powerfully solvable presentations where $0 \leq x \leq n / 2$. Then $\mathcal{P}_{n}=\mathcal{P}(n, 0) \cup \mathcal{P}(n, 1) \cup \cdots \cup \mathcal{P}(n,\lfloor n / 2\rfloor)$ and thus

$$
p^{\alpha n^{3}+o\left(n^{3}\right)}=|\mathcal{P}(n, x(n))| \leq\left|\mathcal{P}_{n}\right|=|\mathcal{P}(n, 0)|+\cdots+|\mathcal{P}(n,\lfloor n / 2\rfloor)| \leq n|\mathcal{P}(n, x(n))|=p^{\alpha n^{3}+o\left(n^{3}\right)} .
$$

This shows that $\left|\mathcal{P}_{n}\right|=p^{\alpha n^{3}+o\left(n^{3}\right)}$. Let us show that this is also the growth of powerfully solvable groups of exponent $p^{2}$ with respect to the order $p^{n}$. Clearly $p^{\alpha n^{3}+o\left(n^{3}\right)}$ gives us an upper bound. We want to show that this is also a lower bound. Let $x=x(n)$ be as above and let $a_{1}, \ldots, a_{n-x}$ be a set of generators for a powerfully solvable group $G$ where $a_{1}^{p}=\cdots=a_{n-2 x}^{p}=1$ and $a_{n-2 x+1}^{p^{2}}=\cdots=a_{n-x}^{p^{2}}=1$. Notice that $\left\langle a_{1}, \ldots, a_{n-2 x}\right\rangle G^{p}=\Omega_{1}(G)$, which is a characteristic subgroup of $G$. It will be useful to consider a larger class of presentations for powerfully solvable groups of order $p^{n}$ where we still require $a_{1}^{p}=\cdots=a_{n-2 x}^{p}=1$ and $a_{n-2 x+1}^{p^{2}}=\cdots=a_{n-x}^{p^{2}}=1$. 
We let $\mathcal{Q}(n, x)=\mathcal{Q}(n, x(n))$ be the collection of all presentations with additional commutator relations

$$
\left[a_{i}, a_{j}\right]=a_{1}^{p \alpha_{1}(i, j)} \cdots a_{n-x}^{p \alpha_{n-x}(i, j)} .
$$

The presentation is included in $\mathcal{Q}(n, x)$ provided the resulting group is powerfully solvable of order $p^{n}$. Notice that $G^{p} \leq Z(G)$ and as a result the commutator relations above only depend on the cosets $\overline{a_{1}}=a_{1} G^{p}, \ldots, \overline{a_{n-x}}=a_{n-x} G^{p}$ and not on the exact values of $a_{1}, \ldots, a_{n-x}$. Consider the vector space $V=G / G^{p}$ over $\mathbb{F}_{p}$ and let $W=\mathbb{F}_{p} \overline{a_{1}}+\cdots+\mathbb{F}_{p} \overline{a_{n-2 x}}$. Then let

$$
H=\{\phi \in \mathrm{GL}(n-x, p): \phi(W)=W\} .
$$

There is now a natural action from $H$ on $\mathcal{Q}(n, x)$. Suppose we have some presentation with generators $a_{1}, \ldots, a_{n-x}$ as above. Let $\phi \in H$ and suppose

$$
{\overline{a_{i}}}^{\phi}=\beta_{1}(i) \overline{a_{1}}+\cdots+\beta_{n-x}(i) \overline{a_{n-x}} .
$$

We then get a new presentation in $\mathcal{Q}(n, x)$ for $G$ with respect to the generators $b_{1}, \ldots, b_{n-x}$ where $b_{i}=a_{1}^{\beta_{1}(i)} \cdots a_{n-x}^{\beta_{n-x}(i)}$.

Suppose there are $l$ powerfully solvable groups of exponent $p^{2}$ and order $p^{n}$ where furthermore $\left|G^{p}\right|=p^{x}$. Pick powerfully solvable presentations $p_{1}, \ldots, p_{l} \in \mathcal{P}(n, x)$ for these. Let $q$ be powerfully solvable presentation in $\mathcal{P}(n, x)$ of a group $K$ with generators $b_{1}, \ldots, b_{n-x}$. Then $q$ is also a presentation for an isomorphic group $G$ with presentation $p_{i}$ and generators $a_{1}, \ldots, a_{n-x}$. Let $\phi: K \rightarrow G$ be an isomorphism and let $\psi: K / K^{p} \rightarrow G / G^{p}$ be the corresponding linear isomorphism. This gives us a linear automorphism $\tau \in H$ induced by $\tau\left(\overline{a_{i}}\right)=\psi\left(\overline{b_{i}}\right)$. Thus $q=p_{i}^{\tau}$. Therefore

$$
\mathcal{P}(n, x) \subseteq p_{1}^{H} \cup p_{2}^{H} \cup \cdots \cup p_{l}^{H} .
$$

From this we get

$$
p^{\alpha n^{3}+o\left(n^{3}\right)}=|\mathcal{P}(n, x)| \leq\left|p_{1}^{H}\right|+\cdots+\left|p_{l}^{H}\right| \leq l p^{n^{2}},
$$

and it follows that $l \geq p^{\alpha n^{3}+o\left(n^{3}\right)}$. We thus get the following result.

Theorem 5.1. The number of powerfully solvable groups of exponent $p^{2}$ and order $p^{n}$ is $p^{\alpha n^{3}+o\left(n^{3}\right)}$, where $\alpha=\frac{-1+\sqrt{2}}{6}$.

As mentioned in [5] the growth of all powerful $p$-groups of exponent $p^{2}$ and order $p^{n}$ is $p^{\frac{2}{27} n^{3}+o\left(n^{3}\right)}$. This claim was though not proved and we will fill in the details here.

As before we consider a group $G$ of order $p^{n}=p^{y+2 x}$ with generators $a_{1}, \ldots, a_{y+x}$ where $o\left(a_{1}\right)=$ $\cdots=o\left(a_{y}\right)=p$ and $o\left(a_{y+1}\right)=\cdots=o\left(a_{y+x}\right)=p^{2}$. This time we can though include all powerful relations

$$
\left[a_{j}, a_{i}\right]=a_{1}^{p \alpha_{y+1}(i, j)} \cdots a_{y+x}^{p \alpha_{y+x}(i, j)}
$$

for $1 \leq i<j \leq y+x$, where $0 \leq \alpha_{k}(i, j) \leq p-1$ for $k=y+1, \ldots, y+x$. For each such pair $(i, j)$ there are $p^{x}$ possible relations for $\left[a_{j}, a_{i}\right]$. We thus see that the number of presentations is $p^{h(x)}$ where

$$
h(x)=\left(\begin{array}{c}
y+x \\
2
\end{array}\right) x=\left(\begin{array}{c}
n-x \\
2
\end{array}\right) x=\frac{x^{3}}{2}-\frac{(2 n-1)}{2} x^{2}+\frac{n(n-1)}{2} x .
$$

Thus

$$
h^{\prime}(x)=\frac{3}{2}\left(x^{2}-\frac{2(2 n-1)}{3} x+\frac{n(n-1)}{3}\right)
$$


and using the same kind of analysis as before we see that for a large $n, h$ takes its maximal value for $x(n)=\frac{2 n-1}{3}-\sqrt{\frac{n^{2}}{9}-\frac{n}{9}+\frac{1}{9}}$. Notice that $\lim _{n \rightarrow \infty} \frac{x(n)}{n}=1 / 3$. Therefore

$$
\lim _{n \rightarrow \infty} \frac{h(x(n)}{n^{3}}=\lim _{n \rightarrow \infty} \frac{1}{2} \cdot\left(\frac{n-x(n)}{n}\right) \cdot\left(\frac{n-1-x(n)}{n}\right) \cdot \frac{x(n)}{n}=2 / 27 .
$$

The same argument as above shows then that the growth of all powerful groups of exponent $p^{2}$ with respect to order $p^{n}$ is $p^{\frac{2}{27} n^{3}+o\left(n^{3}\right)}$.

Later on we will be working with a special subclass $\mathcal{P}$ of powerful $p$-groups, namely those that are of type $(2, r, r, 2)$ with $r \geq 1$. In this case the number of presentations for groups of order $p^{n}, n$ even, is $p^{h(n)}$ where $h(n)=\left(\begin{array}{c}n / 2 \\ 2\end{array}\right) n / 2$ and

$$
\lim _{n \rightarrow \infty} \frac{h(n)}{n^{3}}=\lim _{n \rightarrow \infty} \frac{n / 2(n / 2-1) n / 2}{2 n^{3}}=1 / 16 .
$$

Thus the growth here is $p^{\frac{1}{16} n^{3}+o\left(n^{3}\right)}$.

\section{GROUPS OF TYPE $(2, \ldots, 2)$}

We have seen that powerful nilpotence and powerful solvability is preserved under taking quotients. These properties however work badly under taking subgroups. Our next two results underscore this.

Proposition 6.1. Let $G$ be any powerful p-group of exponent $p^{2}$. There exists a powerfully nilpotent group $H$ of exponent $p^{2}$ and powerful class 2 such that $G$ is powerfully embedded in $H$.

Proof Suppose $G=\left\langle a_{1}, \ldots, a_{r}\right\rangle$ where $a_{1}^{p}=\cdots=a_{s}^{p}=1, a_{s+1}^{p^{2}}=\cdots=a_{r}^{p^{2}}=1$ and where $|G|=p^{s+2(r-s)}$. Let $N=\left\langle x_{s+1}\right\rangle \times \cdots \times\left\langle x_{r}\right\rangle$ be a direct product of cyclic groups of order $p^{2}$. Let $H=(G \times N) / M$, where $M=\left\langle a_{s+1}^{p} x_{s+1}^{-p}, \ldots, a_{r}^{p} x_{r}^{-p}\right\rangle$. Notice that $[G, H]=[G, G] \leq G^{p}$ and thus $G$ is powerfully embedded in $H$. Also, as $[H, H]=G^{p}=N^{p}$, we see that

$$
1 \leq\left\langle x_{1}, \ldots, x_{r}\right\rangle \leq H
$$

is a powerfully central chain and thus $H$ is powerfully nilpotent of powerful class at most 2 .

Remark. (1) There exist powerful $p$-groups of exponent $p^{2}$ that are not powerfully solvable and thus a powerfully embedded subgroup of a powerfully nilpotent group of powerful class 2 does not even need to be powerfully solvable.

(2) There exist powerfully nilpotent groups of exponent $p^{2}$ that are of arbitrary large powerful class and so the proposition above shows that a powerfully nilpotent group of powerful class 2 could have a powerfully embedded powerfully nilpotent subgroup of arbitrary large powerful class.

Next result shows that the subgroup structure of a powerfully nilpotent group of powerful class 2 is even more arbitrary. Notice that such a group is in particular nilpotent of class 2 and it turns out that any finite $p$-group of class 2 can occur as a subgroup.

Proposition 6.2. Let $G$ be any finite p-group of nilpotency class 2. There exists a powerfully nilpotent group $H$ of powerful class 2 that contains $G$ as a subgroup.

Proof Suppose $[G, G]$ has a basis $a_{1}, \ldots, a_{m}$ as an abelian group where $o\left(a_{i}\right)=p^{j_{i}}$. Let $N=$ $\left\langle x_{1}\right\rangle \times \cdots \times\left\langle x_{m}\right\rangle$ be a direct product of cyclic groups where $o\left(x_{i}\right)=p^{j_{i}+1}$. Now let $H=(G \times N) / M$ where $M=\left\langle a_{1} x_{1}^{-p}, \ldots, a_{m} x_{m}^{-p}\right\rangle$. Then $G$ embeds as a subgroup of $H$. Notice also that

$$
1 \leq\left\langle x_{1}, \ldots, x_{m}\right\rangle \leq H
$$


is powerfully central and thus $H$ is powerfully nilpotent of powerful class 2 .

Thus powerful nilpotence and powerful solvability are in general not as satisfactory as notions for powerful groups as nilpotence and solvability for the class of all groups. For a rich subclass of powerful groups things however turn out much better. This is the class $\mathcal{P}$ of all powerful groups of type $(2, \ldots, 2)$ that we considered in Section 5.

For a group $G \in \mathcal{P}$ we have that $G^{p} \leq Z(G)$. It follows that the map $G / G^{p} \rightarrow G^{p}, a G^{p} \mapsto a^{p}$ is a bijection and therefore, for any $H \geq G^{p}$, we have $\left|H / G^{p}\right|=\left|H^{p}\right|$.

Lemma 6.3. Let $G \in \mathcal{P}$ and $H, K \leq G$ where $G^{p} \leq K$. Then $H^{p} \cap K^{p}=(H \cap K)^{p}$.

Proof We have

$$
\begin{aligned}
\left|H^{p} \cap K^{p}\right|=\left|\left(H G^{p}\right)^{p} \cap K^{p}\right| & =\frac{\left|\left(H G^{p}\right)^{p}\right| \cdot\left|K^{p}\right|}{\left|(H K)^{p}\right|} \\
& =\frac{\left|H G^{p} / G^{p}\right| \cdot\left|K / G^{p}\right|}{\left|H K / G^{p}\right|} \\
& =\left|\left(H G^{p} \cap K\right) / G^{p}\right| \\
& =\left|(H \cap K) G^{p} / G^{p}\right| \\
& =\left|(H \cap K)^{p}\right| .
\end{aligned}
$$

As $(H \cap K)^{p} \leq H^{p} \cap K^{p}$ it follows that $H^{p} \cap K^{p}=(H \cap K)^{p}$.

Theorem 6.4. Let $\boldsymbol{G}$ be a powerfully nilpotent group in $\mathcal{P}$ and let $\boldsymbol{H}$ be a powerful subgroup of $G$. Then $H$ is powerfully nilpotent of powerful class less than or equal to the powerful class of $G$.

Proof Suppose $G$ has powerful nilpotence class $c$ and that we have a powerfully central chain $G=G_{0}>G_{1}>\cdots>G_{c}=1$. As $G^{p} \leq Z(G)$ and $\left(G^{p}\right)^{p}=1$, multiplying a term by $G^{p}$ makes no difference. Also as the powerful class is $c$ we get a strictly decreasing powerfully central chain $G=G_{0}>G_{1} G^{p}>\cdots>G_{c-1} G^{p}>1$. Without loss of generality we can thus assume that $G_{1}, \ldots, G_{c-1}$ contain $G^{p}$ as a subgroup. We claim that

$$
H=H \cap G_{0} \geq H \cap G_{1} \geq \cdots \geq H \cap G_{c-1} \geq 1
$$

is powerfully central. Using Lemma 6.3 we have

$$
\left[H \cap G_{i}, H\right] \leq[H, H] \cap\left[G_{i}, G\right] \leq H^{p} \cap G_{i+1}^{p}=\left(H \cap G_{i+1}\right)^{p},
$$

for $0 \leq i \leq c-1$. Hence $H$ is powerfully nilpotent of powerful class at most $c$.

Theorem 6.5. Let $\boldsymbol{G}$ be a powerfully solvable group in $\mathcal{P}$ and let $H$ be a powerful subgroup of $G$. Then $H$ is powerfully solvable of powerful derived length less than or equal to the powerful derived length of $G$.

Proof Suppose the powerful derived length of $G$ is $d$ and that we have a powerfully abelian chain $G=G_{0}>G_{1}>\cdots>G_{d}=1$. Arguing like in the proof of the previous theorem, we can assume that $G_{d-1}$ contains $G^{p}$. We show that

$$
H=H \cap G_{0} \geq H \cap G_{1} \geq \cdots \geq H \cap G_{c-1} \geq H \cap G_{c}=1
$$

is a powerfully abelian chain. Using Lemma 6.3, we have $\left[H \cap G_{i}, H \cap G_{i}\right] \leq[H, H] \cap\left[G_{i}, G_{i}\right] \leq$ $H^{p} \cap G_{i+1}^{p}=\left(H \cap G_{i+1}\right)^{p}$. This shows that $H$ is powerfully solvable of powerful derived length at most $d$. 
We introduce some useful notation. We use $H \leq_{\mathcal{P}} G$ to stand for $H, G \in \mathcal{P}$ and $H \leq G$. We use $H \unlhd_{\mathcal{P}} G$ for $H, G \in \mathcal{P}$ and $H$ powerfully embedded in $G$. The notations $H<_{\mathcal{P}} G$ and $H \triangleleft_{\mathcal{p}} G$ are defined naturally in a similar way.

Let $G$ be a powerful $p$-group in $\mathcal{P}$ and let $V=G / G^{p}$ be the associated vector space over $\mathbb{F}_{p}$. The structure of $G$ is determined by the commutator relations

$$
[a, b]=c^{p},
$$

where there exists such $c \in G$ for each pair $a, b$ in $G$. Notice that $[a, b]$ and $c^{p}$ only depend on the cosets $a G^{p}, b G^{p}$ and $c G^{p}$. Identifying the two vector spaces $G / G^{p}$ and $G^{p}$ under the map $G / G^{p} \rightarrow G^{p}, x G^{p} \mapsto x^{p}$, we get a natural alternating product on $V$ with the relations (1) translating to

$$
\left[a G^{p}, b G^{p}\right]=c G^{p} .
$$

Let $\mathcal{G}$ be the collection of all powerful subgroups of $G$ that are of type $(2, \ldots, 2)$ and let $\mathcal{V}$ be the collection of all the alternating subalgebras of $V$. So for $U$ to be a subalgebra of $V$ it needs to be a subspace where $[U, U] \leq U$. Notice that $[H, H] \leq H^{p}$ translates to $\left[H G^{p} / G^{p}, H G^{p} / G^{p}\right] \leq$ $H G^{p} / G^{p}$. Recall that for $H, K \in \mathcal{G}$ we write $H \unlhd_{\mathcal{P}} K$ for $H$ powerfully embedded in $K$. For $U, W \in \mathcal{V}$ we likewise write $U \unlhd W$ for $U$ an ideal of $W$. Notice that $[H, K] \leq H^{p}$ translates to $\left[H G^{p} / G^{p}, K G^{p} / G^{p}\right] \leq H G^{p} / G^{p}$.

If $H, G \in \mathcal{P}$ such that $G$ is powerfully nilpotent and $H \unlhd_{\mathcal{P}} G$, then the quotient $G / H$ has naturally the structure of powerful group of type $(2, \ldots, 2)$ with $[a H, b H]=[a, b] H$.

Definition. We say that a group $G \in \mathcal{P}$ is powerfully simple if $G \neq 1$ and if $H \triangleleft_{\mathcal{P}} G$ implies that $H=1$.

Definition. Let $H, G \in \mathcal{P}$ with $H \triangleleft_{\mathcal{P}} G$. We say that $H$ is a maximal powerfully embedded $\mathcal{P}$-subgroup of $G$ if there is no $H<K<G$ such that $K \unlhd_{\mathcal{P}} G$.

Lemma 6.6. Let $G \in \mathcal{P}$. We have that $H$ is a maximal powerfully embedded $\mathcal{P}$-subgroup of $G$ if and only if $\mathrm{G} / \mathrm{H}$ is powerfully simple.

Proof Let $H<K<G$. Now as $H$ is powerful of type $(2, \ldots, 2)$ we have $H \cap G^{p}=H^{p}$. Therefore $[K, G] \leq K^{p} H$ if and only if

$$
[K, G] \leq\left(K^{p} H\right) \cap G^{p}=K^{p}\left(H \cap G^{p}\right)=K^{p} H^{p}=K^{p} .
$$

The result follows from this.

Remark. Let $H, K \in \mathcal{G}$ and let $U$ and $W$ be the associated alternating algebras in $\mathcal{V}$. Suppose that $H$ is powerfully embedded in $K$. Then $K / H$ is powerfully simple if and only if $W / U$ is a simple alternating algebra and the latter happens if and only if $U$ is a maximal ideal of $V$.

We will next prove a Jordan-Holder type theorem for alternating algebras. Suppose $A, B, a, b \in$ $\mathcal{V}$ where $A \triangleleft B$ and $a \triangleleft b$. Let $\mathcal{I}_{A}^{B}=\{Z: A \leq Z \leq B\}$ and $\mathcal{I}_{a}^{b}=\{x: a \leq x \leq b\}$. We get natural projections $P: \mathcal{I}_{a}^{b} \rightarrow \mathcal{I}_{A}^{B}$ and $Q: \mathcal{I}_{A}^{B} \rightarrow \mathcal{I}_{a}^{b}$ given by

$$
P(x)=A+B \cap x \text { and } Q(Z)=a+b \cap Z .
$$

Lemma 6.7. We have $P(a) \unlhd P(b)$ and $Q(A) \unlhd Q(B)$. Furthermore $P(b) / P(a)$ is isomorphic to $Q(B) / Q(A)$.

Proof Notice that $P(a)=A+B \cap a, P(b)=A+B \cap b, Q(A)=a+b \cap A$ and $Q(B)=a+b \cap B$. As $A \unlhd B$, we have $[P(a), P(b)]=[A+B \cap a, A+B \cap b] \leq A+[B \cap a, B \cap b]$. Now as $B$ is a subalgebra and $a \unlhd b$ we have that this is contained in $A+B \cap a$. The second claim follows 
from this by symmetry.

Now for $P(b) / P(a)$, notice first that we have

$$
B \cap b \cap(A+B \cap a)=B \cap b \cap A+B \cap a=A \cap b+B \cap a,
$$

and for $u, v, w \in B \cap b$ it follows that

$$
[u, v]+A+B \cap a=w+A+B \cap a \Leftrightarrow[u, v]+A \cap b+B \cap a=w+A \cap b+B \cap a .
$$

By symmetry

$$
[u, v]+a+b \cap A=w+a+b \cap A \Leftrightarrow[u, v]+a \cap B+b \cap A=w+A \cap b+B \cap a .
$$

The isomorphism of $P(b) / P(a)$ and $P(B) / B(A)$ follows from this.

The Jordan-Holder theorem for alternating algebras is proved from this in the standard way.

Definition. Let $V$ be an alternating algebra. A chain $0=U_{0} \triangleleft U_{1} \triangleleft \cdots \triangleleft U_{n}=V$ is a composition series for $V$ if all the factors $U_{1} / U_{0}, \ldots, U_{n} / U_{n-1}$ are simple alternating algebras.

Theorem 6.8. Let $V$ be an alternating algebra. Then all composition series have the same length and same composition factors up to order.

Definition. Let $G \in \mathcal{P}$. A chain $1=H_{0} \triangleleft_{\mathcal{P}} H_{1} \triangleleft_{\mathcal{P}} \cdots \triangleleft_{\mathcal{P}} H_{n}=G$ is a powerful composition series for $G$ if all the factors $H_{1} / H_{0}, \ldots, H_{n} / H_{n-1}$ are powerfully simple.

Theorem 6.9. Let $G$ be a powerful p-group of type $(2, \ldots, 2)$ with two powerful composition series, say

$$
1=H_{0} \triangleleft_{\mathcal{P}} H_{1} \triangleleft_{\mathcal{P}} \cdots \triangleleft_{\mathcal{P}} H_{n}=G
$$

and

$$
1=K_{0} \triangleleft_{\mathcal{P}} K_{1} \triangleleft_{\mathcal{P}} \cdots \triangleleft_{\mathcal{P}} K_{m}=G .
$$

Then $m=n$ and the powerfully simple factors $H_{1} / H_{0}, H_{2} / H_{1}, \ldots, H_{n} / H_{n-1}$ are isomorphic to $K_{1} / K_{0}, K_{2} / K_{1}, \ldots, K_{n} / K_{n-1}$ (in some order).

Proof Replace the terms $H_{i}, K_{j}$ by their associated alternating algebras $U_{i}, V_{j}$. The result now follows from the Jordan-Holder theorem for alternating algebras.

Definition. We refer to the unique factors of a powerful composition series of a group $G \in \mathcal{P}$ as the powerful composition factors of $G$.

Corollary 6.10. A group $G \in \mathcal{P}$ is powerfully solvable if and only if the powerful composition factors are cyclic of order $p^{2}$.

Proof Any powerful abelian chain of $G$ can be refined to a chain with factors that are cyclic of order $p^{2}$.

\section{THE CLASSIFICATION OF POWERFULly SIMPLE GROUPS OF TYPE $(2,2,2)$.}

From previous section we know that this task is equivalent to classifying all simple alternating algebras of dimension 3 .

Following [4], any given alternating algebra of dimension 3 over $\mathbb{F}_{p}$ can be represented by a $3 \times 3$ matrix over $\mathbb{F}_{p}$. Here the matrix

$$
\left[\begin{array}{lll}
\alpha_{11} & \alpha_{12} & \alpha_{13} \\
\alpha_{21} & \alpha_{22} & \alpha_{23} \\
\alpha_{31} & \alpha_{32} & \alpha_{33}
\end{array}\right]
$$


corresponds to the 3 -dimensional alternating algebra $\mathbb{F}_{p} v_{1}+\mathbb{F}_{p} v_{2}+\mathbb{F}_{p} v_{3}$ where

$$
\begin{aligned}
& v_{2} v_{3}=\alpha_{11} v_{1}+\alpha_{21} v_{2}+\alpha_{31} v_{3} \\
& v_{3} v_{1}=\alpha_{12} v_{1}+\alpha_{22} v_{2}+\alpha_{32} v_{3} \\
& v_{1} v_{2}=\alpha_{13} v_{1}+\alpha_{23} v_{2}+\alpha_{33} v_{3} .
\end{aligned}
$$

From last section we know that this corresponds to a powerful $p$-group of order $p^{6}$ with generators $a_{1}, a_{2}, a_{3}$ of order $p^{2}$ satisfying the relations:

$$
\begin{aligned}
& {\left[a_{2}, a_{3}\right]=a_{1}^{p \alpha_{11}} a_{2}^{p \alpha_{21}} a_{3}^{p \alpha_{31}}} \\
& {\left[a_{3}, a_{1}\right]=a_{1}^{p \alpha_{12}} a_{2}^{p \alpha_{22}} a_{3}^{p \alpha_{32}}} \\
& {\left[a_{1}, a_{2}\right]=a_{1}^{p \alpha_{13}} a_{2}^{p \alpha_{23}} a_{3}^{p \alpha_{33}} .}
\end{aligned}
$$

In [4] it is shown that two such matrices $A, B$ represent the same alternating algebra with respect to different basis if and only there exists an invertible $3 \times 3$ matrix $P$ such that

$$
B=\frac{1}{\operatorname{det}(P)} P^{t} A P \text {. }
$$

We write $B \simeq A$ if they are related in this way. This turns out to be slightly more general than being congruent (that is $B=P^{t} A P$ ).

Lemma 7.1. Let $\lambda \in \mathbb{F}_{p}^{*}$. Then $\lambda A \simeq A$.

Proof Let $P=\frac{1}{\lambda} I$. Then $\frac{1}{\operatorname{det}(P)} P^{t} A P=\lambda^{3} \cdot \frac{1}{\lambda^{2}} A=\lambda A$.

From this we easily get the following corollary.

Proposition 7.2. We have $B \simeq A$ if and only if there exists $C$ such that $A$ is congruent to $C$ and $B=\lambda C$.

In particular two matrices that are congruent are equivalent. We can write each such matrix $A$ in a unique way as a sum of a symmetric and an anti-symmetric matrix, namely $A_{s}=\frac{A+A^{t}}{2}$ and $A_{a}=\frac{A-A^{t}}{2}$. As shown in [4] we have that $A \simeq B$ if and only if $A_{s} \simeq B_{s}$ and $A_{a} \simeq B_{a}$. We will determine all the equivalence classes and therefore all powerful $p$-groups of type $(2,2,2)$. From this we will then single out those that are powerfully simple.

Classification of the symmetric matrices. It is known that every symmetric matrix is congruent to a diagonal matrix and furthermore to exactly one of the following $D(1,1,1), D(\tau, 1,1)$, $D(1,1,0), D(\tau, 1,0), D(1,0,0), D(\tau, 0,0)$ and $D(0,0,0)$, where $\tau$ is a fixed non-square in $\mathbb{F}_{p}^{*}$ and $D(\alpha, \beta, \gamma)$ is the $3 \times 3$ matrix with $\alpha, \beta$ and $\gamma$ on the diagonal (compare [1, Chapter 6 , Theorem 2.7]). Now $D(1,1,1)$ is equivalent to $\tau D(1,1,1)$ where the latter has determinant $\tau$ modulo $\left(\mathbb{F}_{p}^{*}\right)^{2}$. Hence $D(1,1,1)$ and $D(\tau, 1,1)$ are equivalent. Also $D(\tau, 0,0)=\tau D(1,0,0)$ is equivalent $D(1,0,0)$. When the rank is 2 then multiplying the matrix by a constant $\lambda \in \mathbb{F}_{p}^{*}$ doesn't change the value of the determinant modulo $\left(\mathbb{F}_{p}^{*}\right)^{2}$. Hence $D(1,1,0)$ and $D(\tau, 1,0)$ are not equivalent. Up to equivalence we thus get only 5 matrices:

$$
D(1,1,1), D(1,1,0), D(\tau, 1,0), D(1,0,0) \text { and } D(0,0,0) \text {. }
$$

Classification of the anti-symmetric matrices. The situation regarding the anti-symmetric matrices is simpler as there are only two equivalence classes. One containing the zero matrix and one for the non-zero matrices. This comes from the fact that there are only two alternating forms (up to isomorphism) for a 3-dimensional algebra $V$. Either $V^{\perp}$ has dimension 3 or 1 .

Classification of the alternating algebras. Let $A$ be some $3 \times 3$ matrix over $\mathbb{F}_{p}$ and let $V$ be 
the corresponding alternating algebra. The symmetric part of $A$ equips $V$ with a corresponding symmetric bilinear form $\langle,\rangle_{s}$ and the anti-symmetric part of $A$ equips $V$ with a corresponding alternating form $\langle,\rangle_{a}$. Now there are two possibilities for $\langle,\rangle_{a}$. If it is zero then $A$ is symmetric and we get five alternating algebras corresponding to the 5 diagonal matrices listed above. From now on we can thus assume that $\langle,\rangle_{a}$ is non-zero. Thus $V^{\perp_{a}}$ is of dimension 1. Say

$$
V^{\perp_{a}}=\mathbb{F}_{p} v_{3}
$$

so that

$$
V=\left(\mathbb{F}_{p} v_{1}+\mathbb{F}_{p} v_{2}\right) \mathbb{\perp}_{a} \mathbb{F}_{p} v_{3}
$$

for some $v_{1}, v_{2} \in V$. For our classification we will divide first into 3 cases. For Case 1 , we have $\left\langle v_{3}, v_{3}\right\rangle_{s} \neq 0$. For Case 2, we have $\left\langle v_{3}, v_{3}\right\rangle_{s}=0$ and $\left(V^{\perp_{a}}\right)^{\perp_{s}}=\left(\mathbb{F}_{p} v_{3}\right)^{\perp_{s}}=V$. Finally for Case 3 , we have $\left\langle v_{3}, v_{3}\right\rangle_{s}=0$ and $\left(V^{\perp_{a}}\right)^{\perp_{s}}=\left(\mathbb{F}_{p} v_{3}\right)^{\perp_{s}}<V$.

Case 1. We can here find a basis $v_{1}, v_{2}, v_{3}$ for $V$ where

$$
V=\mathbb{F}_{p} v_{1} \oplus_{s} \mathbb{F}_{p} v_{2} \oplus_{s} \mathbb{F}_{p} v_{3} \text {. }
$$

Case 1.1. Suppose first that the rank of $\langle,\rangle_{s}$ is 1 . In this case it is easy to see that we can pick our basis further so that

$$
\begin{aligned}
& \left\langle v_{1}, v_{2}\right\rangle_{a}=1, \quad\left\langle v_{1}, v_{3}\right\rangle_{a}=0, \quad\left\langle v_{2}, v_{3}\right\rangle_{a}=0 \\
& \left\langle v_{1}, v_{1}\right\rangle_{s}=0, \quad\left\langle v_{2}, v_{2}\right\rangle_{s}=0, \quad\left\langle v_{3}, v_{3}\right\rangle_{s}=1 .
\end{aligned}
$$

Indeed, notice that we can always multiply the relevant matrix by a constant to get $\left\langle v_{3}, v_{3}\right\rangle_{s}=1$ and then it is easy to pick our $v_{1}, v_{2}$ such that $\left\langle v_{1}, v_{2}\right\rangle_{a}=1$. In this case we thus have only 1 algebra.

Case 1.2. Suppose next that the rank of $\langle,\rangle_{s}$ is 2 . Here again by multiplying by a constant we can assume that $\left\langle v_{3}, v_{3}\right\rangle_{s}=1$ and we can assume that $\left\langle v_{2}, v_{2}\right\rangle_{s}=0$. Now $\left\langle v_{1}, v_{1}\right\rangle_{s}=\lambda^{2}$ or $\left\langle v_{1}, v_{1}\right\rangle_{s}=\tau \lambda^{2}$ for some $\lambda \in \mathbb{F}_{p}^{*}$. By replacing $v_{1}$ by $\frac{1}{\lambda} v_{1}$ we can assume that $\left\langle v_{1}, v_{1}\right\rangle_{s}$ is either 1 or $\tau$. Notice that we have also seen above that these cases are genuinely distinct. Now that $v_{1}$ has been chosen we can replace $v_{2}$ by a suitable multiple to ensure that $\left\langle v_{1}, v_{2}\right\rangle_{a}=1$. We thus get 2 algebras

$$
\begin{aligned}
& \left\langle v_{1}, v_{2}\right\rangle_{a}=1, \quad\left\langle v_{1}, v_{3}\right\rangle_{a}=0, \quad\left\langle v_{2}, v_{3}\right\rangle_{a}=0 \\
& \left\langle v_{1}, v_{1}\right\rangle_{s}=1, \quad\left\langle v_{2}, v_{2}\right\rangle_{s}=0, \quad\left\langle v_{3}, v_{3}\right\rangle_{s}=1 .
\end{aligned}
$$

and

$$
\begin{aligned}
& \left\langle v_{1}, v_{2}\right\rangle_{a}=1, \quad\left\langle v_{1}, v_{3}\right\rangle_{a}=0, \quad\left\langle v_{2}, v_{3}\right\rangle_{a}=0 \\
& \left\langle v_{1}, v_{1}\right\rangle_{s}=\tau, \quad\left\langle v_{2}, v_{2}\right\rangle_{s}=0, \quad\left\langle v_{3}, v_{3}\right\rangle_{s}=1 .
\end{aligned}
$$

Case 1.3. We are then only left with the case where the rank of $\langle,\rangle_{s}$ is 3 . It is not difficult to see that in this case we can pick $v_{1}, v_{2}, v_{3}$ such that

$$
\begin{array}{lll}
\left\langle v_{1}, v_{2}\right\rangle_{a}=1, & \left\langle v_{1}, v_{3}\right\rangle_{a}=0, & \left\langle v_{2}, v_{3}\right\rangle_{a}=0, \\
\left\langle v_{1}, v_{1}\right\rangle_{s}=\alpha, & \left\langle v_{2}, v_{2}\right\rangle_{s}=1, & \left\langle v_{3}, v_{3}\right\rangle_{s}=1,
\end{array}
$$

where $\alpha \in \mathbb{F}_{p}^{*}$. We want to see when we get an equivalent algebra by changing $\alpha$ to $\beta$. If we multiply the presentation by a constant it must be by a square if we still want $\left\langle v_{3}, v_{3}\right\rangle_{s}=1$. Say, we multiply by $\lambda^{2}$ and then replace $v_{3}$ by $\frac{1}{\lambda} v_{3}$. Notice that we now have

$$
\left\langle v_{1}, v_{2}\right\rangle_{a}=\lambda^{2},\left\langle v_{1}, v_{1}\right\rangle_{s}=\alpha \lambda^{2},\left\langle v_{2}, v_{2}\right\rangle_{s}=\lambda^{2}
$$


We are now looking for all possible $\bar{v}_{1}=a v_{1}+b v_{2}$ and $\bar{v}_{2}=c v_{1}+d v_{2}$ where $\left\langle\bar{v}_{1}, \bar{v}_{2}\right\rangle_{a}=1$, $\left\langle\bar{v}_{1}, \bar{v}_{2}\right\rangle_{s}=0$ and $\left\langle\bar{v}_{2}, \bar{v}_{2}\right\rangle_{s}=1$. This gives us the following system of equations:

$$
\begin{aligned}
\lambda^{2}(a d-b c) & =1 \\
\lambda^{2}(\alpha a c+b d) & =0 \\
\lambda^{2}\left(\alpha c^{2}+d^{2}\right) & =1 .
\end{aligned}
$$

We look first for all the solutions where $c=0$. Notice that in this case we must have $\lambda^{2} a d=1$, $\lambda^{2} d^{2}=1$ and $b=0$. Thus $\left\langle\bar{v}_{1}, \bar{v}_{1}\right\rangle_{s}=\lambda^{2}\left(\alpha a^{2}+b^{2}\right)=\lambda^{2} \frac{\alpha}{d^{2} \lambda^{4}}=\frac{\alpha}{\lambda^{2} d^{2}}=\alpha$.

Next we look for solutions where $c \neq 0$ but $d=0$. Then we must have $\lambda^{2} b c=-1, \lambda^{2} \alpha c^{2}=1$ and $a=0$. Here $\left\langle\bar{v}_{1}, \bar{v}_{1}\right\rangle_{s}=\lambda^{2}\left(\alpha a^{2}+b^{2}\right)=\frac{\lambda^{2}}{c^{2} \lambda^{4}}=\frac{\alpha}{\alpha c^{2} \lambda^{2}}=\alpha$.

Finally we are left with finding all solutions where $c d \neq 0$. Then $a=-\frac{b d}{\alpha c}$, and the first equation above gives us

$$
1=-\lambda^{2}\left(\frac{b d^{2}}{\alpha c}+b c\right)=-\frac{b}{\alpha c} \cdot \lambda^{2}\left(d^{2}+\alpha c^{2}\right)=-\frac{b}{\alpha c} .
$$

Thus $b=-\alpha c$ and $a=-\frac{b d}{\alpha c}=d$. Therefore

$$
\begin{aligned}
\left\langle\bar{v}_{1}, \bar{v}_{1}\right\rangle & =\lambda^{2}\left(\alpha a^{2}+b^{2}\right) \\
& =\lambda^{2}\left(\alpha d^{2}+\alpha^{2} c^{2}\right) \\
& =\alpha \lambda^{2}\left(\alpha c^{2}+d^{2}\right) \\
& =\alpha .
\end{aligned}
$$

We have thus seen that the value of $\alpha$ doesn't change and we have $\boldsymbol{p}-\mathbf{1}$ different algebras here.

Case 2. Here we are assuming that $\left\langle v_{3}, v_{3}\right\rangle_{s}=0$ and that $v_{3}$ is orthogonal to $v_{1}, v_{2}$ as well. Again we consider few subcases.

Case 2.1. Suppose that the rank of $\langle,\rangle_{s}$ is zero. Then clearly we have $\mathbf{1}$ algebra.

$$
\begin{aligned}
& \left\langle v_{1}, v_{2}\right\rangle_{a}=1, \quad\left\langle v_{1}, v_{3}\right\rangle_{a}=0, \quad\left\langle v_{2}, v_{3}\right\rangle_{a}=0, \\
& \left\langle v_{1}, v_{1}\right\rangle_{s}=0, \quad\left\langle v_{2}, v_{2}\right\rangle_{s}=0, \quad\left\langle v_{3}, v_{3}\right\rangle_{s}=0 .
\end{aligned}
$$

Case 2.2. Suppose next that the rank of $\langle,\rangle_{s}$ is 1 . By multiplying by a suitable constant we can assume that $\left\langle v_{1}, v_{1}\right\rangle_{s}=1$ and $\left\langle v_{2}, v_{2}\right\rangle_{s}=0$. Finally replacing $v_{2}$ by an appropriate multiple we can also assume that $\left\langle v_{1}, v_{2}\right\rangle_{a}=1$. We thus also get here only $\mathbf{1}$ algebra

$$
\begin{aligned}
& \left\langle v_{1}, v_{2}\right\rangle_{a}=1, \quad\left\langle v_{1}, v_{3}\right\rangle_{a}=0, \quad\left\langle v_{2}, v_{3}\right\rangle_{a}=0, \\
& \left\langle v_{1}, v_{1}\right\rangle_{s}=1, \quad\left\langle v_{2}, v_{2}\right\rangle_{s}=0, \quad\left\langle v_{3}, v_{3}\right\rangle_{s}=0 .
\end{aligned}
$$

Case 2.3. Finally we are left with the case when the rank of $\langle,\rangle_{s}$ is 2 . Here it is easy to see that we can pick our basis further so that

$$
\begin{array}{lll}
\left\langle v_{1}, v_{2}\right\rangle_{a}=1, & \left\langle v_{1}, v_{3}\right\rangle_{a}=0, & \left\langle v_{2}, v_{3}\right\rangle_{a}=0, \\
\left\langle v_{1}, v_{1}\right\rangle_{s}=\alpha, & \left\langle v_{2}, v_{2}\right\rangle_{s}=1, & \left\langle v_{3}, v_{3}\right\rangle_{s}=0 .
\end{array}
$$

Similar calculations as for Case 1.3 show that we get distinct algebras for different values of $\alpha$. Thus here we have $\boldsymbol{p}-\mathbf{1}$ algebras. 
Case 3. Here we are assuming that $\left\langle v_{3}, v_{3}\right\rangle_{s}=0$ but that $v_{3}$ is not orthogonal to everything in $V$ with respect to $\langle,\rangle_{s}$. Thus $\left(\mathbb{F}_{p} v_{3}\right)^{\perp_{s}}$ has dimension 2. Suppose

$$
\left(\mathbb{F}_{p} v_{3}\right)^{\perp_{s}}=\mathbb{F}_{p} v_{2}+\mathbb{F}_{p} v_{3}
$$

It is not difficult to see that we can pick our basis such that

$$
\begin{aligned}
& \left\langle v_{1}, v_{2}\right\rangle_{a}=1, \quad\left\langle v_{1}, v_{3}\right\rangle_{a}=0, \quad\left\langle v_{2}, v_{3}\right\rangle_{a}=0, \\
& \left\langle v_{1}, v_{1}\right\rangle_{s}=0, \quad\left\langle v_{1}, v_{2}\right\rangle_{s}=0, \quad\left\langle v_{1}, v_{3}\right\rangle_{s}=1, \quad\left\langle v_{2}, v_{3}\right\rangle_{s}=0 .
\end{aligned}
$$

Now there are two subcases.

Case 3.1. If the rank of $\langle,\rangle_{s}$ is 2 then we must have $\left\langle v_{2}, v_{2}\right\rangle_{s}=0$ and this gives us $\mathbf{1}$ algebra.

Case 3.2. If the rank of $\langle,\rangle_{s}$ is 3 then $\left\langle v_{2}, v_{2}\right\rangle_{s} \neq 0$ and after multiplying by a suitable constant we can assume that this value is 1 (and then afterwards adjust things so that the other assumptions hold again). Thus we get again 1 algebra.

Adding up we see that in total we get $12+2(p-1)$ algebras and thus the same number of powerful $p$-groups of type $(2,2,2)$. Before listing these we state and prove a proposition that shows how we can see which of these are powerfully simple.

Proposition 7.3. An alternating algebra $V$ over $\mathbb{F}_{p}$ of dimension 3 is simple if and only if $V \cdot V=$ $V$.

Proof This condition is clearly necessary as $V \cdot V$ is an ideal of $V$. To see that it is sufficient, suppose $V \cdot V=V$ and let $I$ be a proper ideal. We want to show that $I=0$. We argue by contradiction and suppose $I$ is an ideal of dimension either 1 or 2 . If $I$ is of dimension 2, then $V / I$ is 1 dimensional and thus we get the contradiction that $V \cdot V \leq I<V$. Now suppose $I$ is of dimension 1 , say $V=I+\mathbb{F}_{p} v_{1}+\mathbb{F}_{p} v_{2}$. Then $V \cdot V \leq I+\mathbb{F}_{p} v_{1} v_{2}$. But the dimension of $I+\mathbb{F} v_{1} v_{2}$ is at most 2 and we get the contradiction that $V \cdot V<V$.

We have thus determined the presentation matrices up to equivalence and got in total $12+2(p-1)$. As we described at the beginning of the section this gives us a classification of all the alternating algebras of dimension 3 over $\mathbb{F}_{p}$ that in turn gives us a classification of all the powerful $p$-groups of type $(2,2,2)$. Furthermore, last proposition tells us how we read from the presentation whether a given alternating algebra is simple and thus whether the corresponding powerful group is powerfully simple. The work above gives us the following list of powerful $p$-groups of type $(2,2,2)$. As the power relations for all of these are $a_{1}^{p^{2}}=a_{2}^{p^{2}}=a_{3}^{p^{2}}=1$ we omit these below. Here $\tau$ is a fixed non-square in $\mathbb{F}_{p}$. 


$$
\begin{aligned}
& A_{1}=\left\langle a_{1}, a_{2}, a_{3}:\left[a_{2}, a_{3}\right]=a_{1}^{p},\left[a_{3}, a_{1}\right]=a_{2}^{p},\left[a_{1}, a_{2}\right]=a_{3}^{p}\right\rangle ; \\
& A_{2}=\left\langle a_{1}, a_{2}, a_{3}:\left[a_{2}, a_{3}\right]=a_{2}^{p},\left[a_{3}, a_{1}\right]=a_{1}^{p},\left[a_{1}, a_{2}\right]=a_{3}^{p}\right\rangle ; \\
& A_{3}=\left\langle a_{1}, a_{2}, a_{3}:\left[a_{2}, a_{3}\right]=a_{1}^{p} a_{2}^{-p},\left[a_{3}, a_{1}\right]=a_{1}^{p},\left[a_{1}, a_{2}\right]=a_{3}^{p}\right\rangle ; \\
& A_{4}=\left\langle a_{1}, a_{2}, a_{3}:\left[a_{2}, a_{3}\right]=a_{1}^{p \tau} a_{2}^{-p},\left[a_{3}, a_{1}\right]=a_{1}^{p},\left[a_{1}, a_{2}\right]=a_{3}^{p}\right\rangle ; \\
& A_{5}=\left\langle a_{1}, a_{2}, a_{3}:\left[a_{2}, a_{3}\right]=a_{2}^{-p} a_{3}^{p},\left[a_{3}, a_{1}\right]=a_{1}^{p} a_{2}^{p},\left[a_{1}, a_{2}\right]=a_{1}^{p}\right\rangle ; \\
& A_{6}(\alpha)=\left\langle a_{1}, a_{2}, a_{3}:\left[a_{2}, a_{3}\right]=a_{1}^{p \alpha} a_{2}^{-p},\left[a_{3}, a_{1}\right]=a_{1}^{p} a_{2}^{p},\left[a_{1}, a_{2}\right]=a_{3}^{p}\right\rangle, 1 \leq \alpha \leq p-2 ; \\
& B_{1}=\left\langle a_{1}, a_{2}, a_{3}:\left[a_{2}, a_{3}\right]=a_{1}^{-p} a_{2}^{-p},\left[a_{3}, a_{1}\right]=a_{1}^{p} a_{2}^{p},\left[a_{1}, a_{2}\right]=a_{3}^{p}\right\rangle ; \\
& B_{2}=\left\langle a_{1}, a_{2}, a_{3}:\left[a_{2}, a_{3}\right]=a_{1}^{p},\left[a_{3}, a_{1}\right]=a_{2}^{p},\left[a_{1}, a_{2}\right]=1\right\rangle ; \\
& B_{3}=\left\langle a_{1}, a_{2}, a_{3}:\left[a_{2}, a_{3}\right]=a_{1}^{p \tau},\left[a_{3}, a_{1}\right]=a_{2}^{p},\left[a_{1}, a_{2}\right]=1\right\rangle ; \\
& B_{4}=\left\langle a_{1}, a_{2}, a_{3}:\left[a_{2}, a_{3}\right]=a_{2}^{-p},\left[a_{3}, a_{1}\right]=a_{1}^{p},\left[a_{1}, a_{2}\right]=1\right\rangle ; \\
& B_{5}=\left\langle a_{1}, a_{2}, a_{3}:\left[a_{2}, a_{3}\right]=a_{1}^{p} a_{2}^{-p},\left[a_{3}, a_{1}\right]=a_{1}^{p},\left[a_{1}, a_{2}\right]=1\right\rangle ; \\
& B_{6}=\left\langle a_{1}, a_{2}, a_{3}:\left[a_{2}, a_{3}\right]=a_{2}^{-p} a_{3}^{p},\left[a_{3}, a_{1}\right]=a_{1}^{p},\left[a_{1}, a_{2}\right]=a_{1}^{p}\right\rangle \\
& B_{7}(\alpha)=\left\langle a_{1}, a_{2}, a_{3}:\left[a_{2}, a_{3}\right]=a_{1}^{p \alpha} a_{2}^{-p},\left[a_{3}, a_{1}\right]=a_{1}^{p} a_{2}^{p},\left[a_{1}, a_{2}\right]=1\right\rangle, 1 \leq \alpha \leq p-2 ; \\
& C_{1}=\left\langle a_{1}, a_{2}, a_{3}:\left[a_{2}, a_{3}\right]=a_{1}^{-p} a_{2}^{-p},\left[a_{3}, a_{1}\right]=a_{1}^{p} a_{2}^{p},\left[a_{1}, a_{2}\right]=1\right\rangle ; \\
& C_{2}=\left\langle a_{1}, a_{2}, a_{3}:\left[a_{2}, a_{3}\right]=a_{1}^{p},\left[a_{3}, a_{1}\right]=1,\left[a_{1}, a_{2}\right]=1\right\rangle ; \\
& D=\left\langle a_{1}, a_{2}, a_{3}:\left[a_{2}, a_{3}\right]=1,\left[a_{3}, a_{1}\right]=1,\left[a_{1}, a_{2}\right]=1\right\rangle .
\end{aligned}
$$

Of these $12+2(p-1)$ groups, the groups $A_{1}, \ldots, A_{6}(\alpha)$ are the powerfully simple groups. There are $5+(p-2)$ of these.

\section{REFERENCES}

[1] P. M. Cohn, Algebra, Vol. 2, 2nd edition, University College London, 1989.

[2] A. Lubotzky and A. Mann, Powerful p-groups, J. Algebra, 105 (1987), 485-505.

[3] A. Shalev, On almost fixed point free automorphisms, J. Algebra, 157 (1993), 271-282.

[4] G. Traustason, Symplectic alternating algebras, Int. J. Algebra and Comp. , 18 (2008), 719-757.

[5] G. Traustason and J. Williams, Powerfully nilpotent groups, J. Algebra, 522 (2019), 80-100.

[6] G. Traustason and J. Williams, Powerfully nilpotent groups of maximal powerful class, Monatshefte für Mathematik, 191(4) (2020), 779-799.

[7] G. Traustason and J. Williams, Powerfully nilpotent groups of rank 2 or small order, J. Group Theory, to appear. 